\title{
FACE RECOGNITION FOR THE AUTOMATIC ATTENDANCE MANAGEMENT SYSTEM
}

\author{
N.Ramya Sri ${ }^{1}$ \\ ${ }^{1}$ Department of Computer Science and Engineering \\ Koneru Lakshmaiah Education Foundation, Vaddeswaram-522502, Guntur, AP, India \\ D.Manasa ${ }^{2}$ \\ ${ }^{2}$ Department of Computer Science and Engineering \\ Koneru Lakshmaiah Education Foundation, Vaddeswaram-522502, Guntur, AP, India
}

\author{
N.Ramya ${ }^{3}$ \\ ${ }^{3}$ Department of Computer Science and Engineering \\ Koneru Lakshmaiah Education Foundation, Vaddeswaram-522502, Guntur, AP, India
}

\author{
Sk.Naveed ${ }^{4}$ \\ ${ }^{4}$ Department of Computer Science and Engineering \\ Koneru Lakshmaiah Education Foundation, Vaddeswaram-522502, Guntur, AP, India
}

Article DOI: $\underline{\text { https://doi.org/10.36713/epra5583 }}$ \begin{abstract}
is to develop a complete Face Recognition system. The system uses Convolutional Neural Networks in order to extract relevant facial features. These features allow to compare faces between them in an efficient way. The system can be trained to recognize a set of people, and to learn in an on-line way, by integrating the new people. Face recognition system is one of the biometric information process its applicability is easier and working range is larger than others. The face recognition is live acquired images without any application field in mind .process utilized in the system are White Balance correction, skin like region segmentation facial feature extraction and face image extraction on a face Candidate. The face one of the easiest ways to distinguish the individual identify each other .Face recognition is a personal identification system that uses personal characteristics of a person to identify the person's identify.
\end{abstract}

ABSTRACT
Face Recognition is a currently developing technology with multiple real- life applications. The goal of this Thesis

KEYWORDS:-Face Recognition, Facial Attendance, Automatic Attendance, Face Detection.

\section{INTRODUCTION}

Face recognition is as old as computer vision, both because of the practical importance of the topic and theoretical interest from cognitive scientists [1]. Despite the fact that other methods of identification (such as fingerprints, or iris scans) can be more accurate, face recognition has always remains a major focus of research because of its noninvasive nature and because it is people's primary method of person identification [2]. Face recognition technology is gradually evolving to a universal biometric solution since it requires virtually zero effort from the user end while compared with other biometric options [3]. Biometric face recognition is basically used in three main domains: time attendance systems and employee management; visitor management systems; and last but not the least authorization systems and access control systems [4].

The enhancement of science and technology leads to make the life more comfortable than older days. The emerging 


\section{EPRA International Journal of Research and Development (IJRD)}

technologies like neutrosophic shortest path [1,2,3,4,5], transportation problem $[6,7,8]$, uncertainty problem $[9,10,11,12,13,14]$, fuzzy shortest path $[15,16,17,18,19]$, PowerShell [20], wireless sensor network $[21,22,23,24,25,26,27,28]$, computer language [29,30], neural network [31], routing [32] making the products more intelligent and self-healing based. The smart city applications like smart water, smart grid, smart parking, smart resource management, etc. are based on IoT and IoE $[33,34,35,36,37]$ technologies. We have the development available to us to enable the organization of our consistently lives and the sharing of significant information with our associates, families and others. Why development is huge in our consistently life. It is a basic contraption that we can't avoid, it has a huge impact in the vast majority of our lives Technology fundamentally handles the instruments, advancements and strategies used to help us with dealing with issues and simply improve our everyday schedules and easier to encounter to a great extent $[38,39]$. Advancement is inevitable in our normal everyday presences [40]. This is in light of the fact that presence without advancement is senseless in the present incredible world. Development, which joins instruments to propel unforeseen development, use and information exchange, has as its basic objective of making tasks easier and the handling of various issues of mankind. Right when development advances and makes our continues with extensively more worthwhile, we should pressure that it is so useful to our lives.

\subsection{THE FACE RECOGNITION}

That being said, this problem is also a really difficult one, and it has not been until recent years that quality results are being obtained [5]. In fact, this problem is usually split into different sub-problems to make it easier to work with, mainly face detection in an image, followed by the face recognition itself [6]. There are also other tasks that can be performed inbetween, such as frontolyzing faces, or extracting additional features from them [7]. Through the years, many algorithms and techniques have been used, such as eigenfaces or Active Shape models. However, the one that is currently mostly used, and providing the best results, These are currently obtaining high quality results, so, after reviewing the current state of art, we decided to focus this project on them [8].

\subsection{GOAL AND IMPLEMENTATION}

Our goal was to create a complete Face Recognition for Attendance management system, capable of working with any kind of images, and to constantly improve itself [9]. This improvement had to be autonomous, and to allow it to better recognize people in it, and to include new ones. On top of that, the time requirements were also an issue, as this recognition must be done as close to real-time as possible. The task of recognizing faces, especially outside of controlled conditions, is an extremely difficult problem [10]. In fact, there have been many approaches throughout the history that have not succeeded. Apart from the variance between pictures of the same face, such as expression, light conditions or facial hair, it is difficult to determine what makes a face recognizable [11]. The main reasons are the good results obtained being really close to the state of art, and the quality of the description. It consists in a 3-step process [12]. First, the face in the image is located and frontolyzed, so that it is looking at the camera. Then, the frontolyzed face is sent through, and a set of relevant features are extracted [13]. Finally, these features are used as attributes to compare pairs of images to determine whether they belong or not to the same person. And Marks the attendance automatically in the excel sheets [14].

\section{LITERATURE REVIEW}

\subsection{A Counterpart Approach to Attendance}

System using OpenCV Technique :In this paper, the idea of technology namely Student Attendance has been implemented .This system automatically detects the student performance and maintains the student's records like attendance and their feedback on the subjects. Therefore the attendance of the student can be made available by recognizing the face. On recognizing, the attendance details and details about the marks of the student is obtained as feedback [2]. Other paper proposed by introduced a real-time computer vision algorithm in automatic attendance management system. The system installed the camera with non-intrusive, which can snap images in the classroom and compared the extracted face from the image of the camera capturing with faces inside the system. Also HAAR CLASSIFIERS used to train the images from the camera capturing. The face snap by the camera capturing will convert to grayscale and do subtraction on the images; then the image is transferred to store on the server and processing later [3].

\section{MOTIVATION OR MATERIAL AND METHODS \\ 4.1 PROPOSED PROPOSAL}

Every institute has its own method to keep record of attendance of students. Some are taking attendance manually using the traditional pen and paper or file based approach but marking attendance is a lengthy process and takes lot of time and effort, especially if it is a lecture with huge number of students. Also dealing with the records of a large number of students often leads to human error. So we are implementing a system to automatically record the students' attendance during lecture hours in a hall or room using facial recognition technology. This project deals with one of the possible solutions.

\subsection{ARCHITECTURE}

The main working principle of the project is that, the video captured data is converted into image to detect and recognize it. Further the recognized image of the student is provided with attendance; else the system marks the database as absent. 


\section{EPRA International Journal of Research and Development (IJRD)

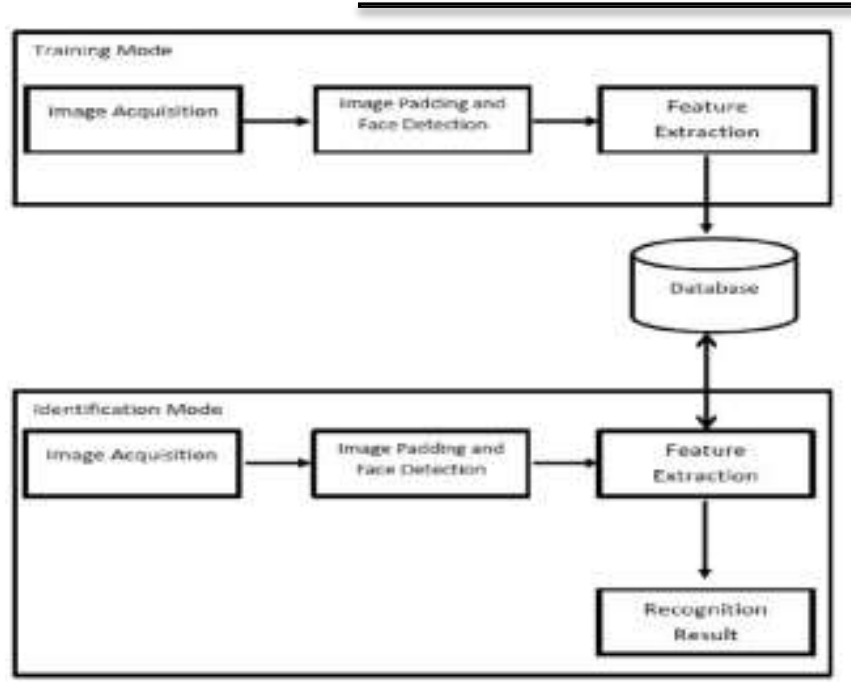

Fig 1. Architecture of proposed model

\subsection{METHODOLOGY}

Our purpose is to make a facial recognition system which needs as less training data as possible. The main reason behind this constraint is the fact that it is more useful for a supervisor to have train the model with one or few pictures for each student rather than having to make a large Dataset with many images for the same person. We will be comparing two main face classification models, PCA dimensionality reduction, and pretrained.

To perform face recognition, the following steps will be followed:

- Detecting all faces included in the image (face detection).

- Cropping the faces and extracting their features.

- Applying a suitable facial recognition algorithm to compare faces with the database of students and lecturers.

- Providing a file recording the identified attendants.

This chapter contains block diagram of face recognition based attendance monitoring system. The elements of block diagram of face recognition based attendance monitoring system using OpenCV are also explained. The process of person identification by using face recognition can be split into three main phases. These are registration and normalization, feature extraction and classification are explained [4]. Our purpose is to make a facial recognition system which needs as less training data as possible. The main reason behind this constraint is the fact that it is more useful for a supervisor to have train the model with one or few pictures for each student rather than having to make a large Dataset with many images for the same person. To perform face recognition the following steps will be followed:

- Detecting all faces included in the image (face detection).

- Cropping the faces and extracting their features.

- Applying a suitable facial recognition algorithm to compare faces with the database of students and lecturers.
- Providing a file recording the identified attendants.

\section{How OpenCV's face recognition works}

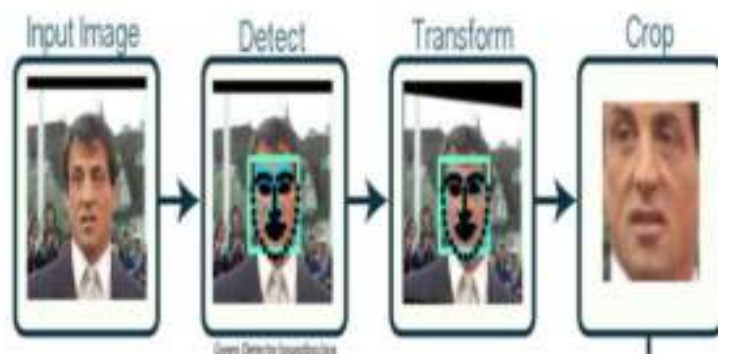

Fig.2 OpenCV Simulation

\section{RESULTS AND DISCUSSION 5.1 FACE DETECTION}

The result of the face Recognition System depends upon the characteristics, position, feature, Extraction and identification of the face image to be analyzed facial recognition process normally has four phases are steps. First Face detection and another one normalization and also feature extraction. Three-dimensional face recognition technique user's 3D sensors to capture information about the shape of a face. A facial recognition system uses biometrics to map facial features from photography or video. In digital camera terminology face detection also called face priority. And a function of a camera that detects human faces.

\subsection{FACIAL RECOGNITION}

Facial recognition is a Biometric software application capable of uniquely identifying or verifying a person by comparing and analyzing patterns based on the persons facial contours. And it is used for security purposes .In face detection I identify Eyes ,Nose ,Mouth .Open CV is the most popular library for Computer Vision .It used for faces Within a Picture and the Faces are complicated .Facial recognition is basically the task of recognition a person Based on its facial image .It has project of Finding Faces. Face recognition Work By identifying facial features by extracting features ,or landmarks From the image of face .For example ,to extract facial features shape and size of the Eyes ,the Size of nose ,and Relative position with Eyes.

\section{SUMMARY AND CONCLUSION}

The enhancement of science and technology leads to make the life more comfortable than older days. The emerging technologies like wireless sensor network $[7,8]$, IoT $[9,10]$, embedded system [11,12] neutrosophic algorithms [13], fuzzy and neural network based algorithms [14], data science, data mining $[15,16]$ making the products more intelligent and selfhealing based. The smart city applications like smart water, smart grid, smart parking, smart resource management, etc. are based on IoT and IoE technologies [17, 18]. We have the development available to us to enable the organization of our consistently lives and the sharing of significant information 


\section{EPRA International Journal of Research and Development (IJRD)}

with our associates, families and others [19, 20]. Why development is huge in our consistently life. It is a basic contraption that we can't avoid, it has a huge impact in the vast majority of our lives Technology fundamentally handles the instruments, advancements and strategies used to help us with dealing with issues and simply improve our everyday schedules and easier to encounter to a great extent [21, 22]. Advancement is inevitable in our normal everyday presences [23]. This is in light of the fact that presence without advancement is senseless in the present incredible world [24]. Development, which joins instruments to propel unforeseen development, use and information exchange, has as its basic objective of making tasks easier and the handling of various issues of mankind. Right when development advances and makes our continues with extensively more worthwhile, we should pressure that it is so useful to our lives.

We have developed a fully working Face Recognition system, with the capacity of on-line learning without human intervention. It can work with any kind of images, and is reasonably robust to changes in face expression or orientation, light conditions and other factors. We have based our face verification step on the deep Face [Taigman et al., 2014] system, which we have used to provide facial features to our FR system. The obtained system has been extensively tested, and different parameters combinations have been tried. We have used the Large Face in the Wild Huang et al., 2007 dataset to assess the performance of our face verification step. Apart from that, we have gathered a 600,000 face images dataset to use both in training and testing. The facial recognition part has been tested using two different sized datasets, and we have obtained steady results around the $90 \%$ of accuracy, reaching a maximum of $95 \%$. These results are better than the ones we expected, and they allow for some real-life use cases. However, there is still room for improvement, as explained in following Section.

Two real applications of the FR technology havealso been presented. The first one is an online web tool that allows for easily training and testing a whole FR system by simply providing sets of images. Even though it is still an alpha version, with limited functionality, it is fully operational and has proven to be useful for demonstration purposes. The other application consists in recognizing people in videos. After processing the video, it draws the bounding box of each person in it, following them around the screen, and writes the name of identified people. It is already working for videos with one person in it, and it is currently being upgraded to allow for multiple people recognition.

\subsection{FUTURE WORK}

Throughout the document we have already pointed out some aspects that should be improved in the future. The main part that needs to be worked on is to enhance the feature extraction part. The currently used is still underperforming compared with the current state of art. As this part is the basis of the whole FR system, any final performance. In order to do so, we plan on gathering more data for training; more thoroughly test the parameters and various configurations, and further experiment with data augmentation. I have developed a fully working Face Recognition system, with the capacity of on-line learning without human intervention. It can work with any kind of images, and is reasonably robust to changes in face expression or orientation, light conditions and other factors.

\section{REFERENCES}

1. Broumi, S., Dey, A., Talea, M. et al. Shortest path problem using Bellman algorithm under neutrosophic environment. Complex \& Intelligent Systems, 5 (2019), 409-416.

2. Kumar, R., Dey, A., Broumi, S., and Smarandache, F. A study of neutrosophic shortest path problem. In Neutrosophic Graph Theory and Algorithms. IGI Global, 2020.

3. Kumar, R., Edalatpanah, S. A., Jha, S., Broumi, S., Singh, R., and Dey, A. A multi objective programming approach to solve integer valued neutrosophic shortest path problems. Neutrosophic Sets and Systems, 24 (2019), 139-151.

4. Kumar, R., Edalatpanah, S. A., Jha, S., and Singh, R. A novel approach to solve gaussian valued neutrosophic shortest path problems. International Journal of Engineering and Advanced Technology, 8 (2019), 347-353.

5. Kumar, R., Edaltpanah, S. A., Jha, S., Broumi, S., and Dey, A. Neutrosophic shortest path problem. Neutrosophic Sets and Systems, 23 (2018), 5-15.

6. Pratihar, J., Kumar, R., Dey, A., and Broumi, S. Transportation problem in neutrosophic environment. In Neutrosophic Graph Theory and Algorithms. IGI Global, 2020.

7. Kumar, R., Edalatpanah, S. A., Jha, S., and Singh, R. A Pythagorean fuzzy approach to the transportation problem. Complex \& Intelligent Systems, 5 (2019), 255-263.

8. Pratihar, J., Kumar, R., Edalatpanah, S. A., and Dey, A. Modified Vogel's approximation method for transportation problem under uncertain environment. Complex \& Intelligent Systems (2020), 1-12.

9. Gayen, S., Jha, S., Singh, M., and Kumar, R. On a generalized notion of anti-fuzzy subgroup and some characterizations. International Journal of Engineering and Advanced Technology, 8 (2019), 385-390.

10. Gayen, S., Smarandache, F., Jha, S., and Kumar, R. Intervalvalued neutrosophic subgroup based on interval-valued triple t-norm. In Neutrosophic Sets in Decision Analysis and Operations Research. IGI Global, 2020.

11. Gayen, S., Smarandache, F., Jha, S., Singh, M. K., Broumi, S., and Kumar, R. Introduction to plithogenic subgroup. In Neutrosophic graph Theory and Algorithms. IGI Global, 2020.

12. Gayen, S., Smarandache, F., Jha, S., Singh, M. K., Broumi, S., and Kumar, R. Soft Subring Theory Under Interval-valued Neutrosophic Environment. Neutrosophic Sets and Systems, 36 (2020), 16.

13. Gayen, S., Smarandache, F., Jha, S., and Kumar, R. Introduction to interval-valued neutrosophic subring. Neutrosophic Sets and Systems, 36 (2020), 17.

14. Gayen, S., Smarandache, F., Jha, S., Singh, M. K., Broumi, S., and Kumar, R. Introduction to plithogenic hypersoft subgroup. Neutrosophic Sets and Systems, 33 (2020), 208-233.

15. Kumar, R., Edalatpanah, S. A., and Mohapatra, H. Note on "Optimal path selection approach for fuzzy reliable shortest path problem “. Journal of Intelligent \& Fuzzy Systems (2020), $1-4$.

16. Kumar, R., Jha, S., and Singh, R. A different approach for solving the shortest path problem under mixed fuzzy environment. International Journal of Fuzzy System 


\section{SJIF Impact Factor: 7.001| ISI I.F.Value:1.241| Journal DOI: 10.36713/epra2016 ISSN: 2455-7838(Online) \\ EPRA International Journal of Research and Development (IJRD)}

Applications, 9 (2020), 132-161.

17. Kumar, R., Jha, S., and Singh, R. Shortest path problem in network with type-2 triangular fuzzy arc length. Journal of Applied Research on Industrial Engineering, 4 (2017), 1-7.

18. Kumar, Ranjan, Edalatpanah, S. A., Jha, Sripati, Gayen, Sudipta, and Singh, Ramayan. Shortest path problems using fuzzy weighted arc length. International Journal of Innovative Technology and Exploring Engineering, 8 (2019), 724-731.

19. Kumar, R., Edalatpanah, S. A., Jha, S., Gayen, S., and Singh, $R$. Shortest path problems using fuzzy weighted arc length. International Journal of Innovative Technology and Exploring Engineering, 8 (2019), 724-731.

20. Mohapatra, H., Panda, S., Rath, A. K., Edalatpanah, S. A., and Kumar, R. A tutorial on powershell pipeline and its loopholes. International Journal of Emerging Trends in Engineering Research, 8 (2020), 975-982.

21. Mohapatra, H., Rath, S., Panda, S., and Kumar, R. Handling of man-in-the-middle attack in wsn through intrusion detection system. International Journal of Emerging Trends in Engineering Research, 8 (2020), 1503-1510.

22. Mohapatra, H., Debnath, S., and Rath, A. K. Energy management in wireless sensor network through eb-leach. International Journal of Research and Analytical Reviews, 56 (2018), 56-61.

23. Mohapatra, H., Rath, A. K., Landge, P. B., Bhise, D., Panda, S., and Gayen, $S$. A comparative analysis of clustering protocols of wireless sensor network. International Journal of Mechanical and Production Engineering Research and Development, 10 (2020), 8371-8386.

24. Mohapatra, H. and Rath, A. K. A survey on fault tolerance based clustering evolution in wsn. IET Networks (2020).

25. Mohapatra, H., Debnath, S., Rath, A. K., Landge, P. B., Gayen, $S$., and Kumar, R. An efficient energy saving scheme through sorting technique for wireless sensor network. International Journal of Emerging Trends in Engineering Research, 8 (2020), 4278-4286.

26. Mohapatra, H. and Rath, A. K. Fault tolerance in wsn through uniform load distribution function. International Journal of Sensors, Wireless Communications and Control, 10 (2020).

27. Mohapatra, H. and Rath, A. K. Fault tolerance through energy balanced cluster formation (ebcf) in wsn. In Smart Innovations in Communication and Computational Sciences ( 2018), springer, 313-321.

28. Mohapatra, Hitesh and Rath, Amiya Kumar. Fault tolerance in WSN through PE-LEACH protocol. IET Wireless Sensor Systems, 9, 6 (Dec. 2019), 358-365(7).

29. Mohapatra, H. C Programming: Practice. Amazon, 2018.

30. Mohapatra, H. and Rath, A. K. Fundamentals of Software Engineering. BPB, 2020.

31. Mohapatra, H. HCR by Using Neural Network. 2009.

32. Panda, M., Pradhan, P., Mohapatra, H., and Barpanda, N. K. Fault tolerant routing in heterogeneous environment. International Journal of Scientific \& Technology Research, 8 (2019), 1009-1013.

33. Mohapatra, H. Offline drone instrumentalized ambulance for emergency situations. International Journal of Robotics and Automation, 9 (2020), 251-255.

34. Mohapatra, H. and Rath, A. K. Detection and avoidance of water loss through municipality taps in india by using smart tap and ict. IET Wireless Sensor Systems (2019), https--doi.
35. Panda, H., Mohapatra, H., and Rath, A. K. Wsn-based water channelization: an approach of smart water. In Smart Cities: Opportunities and Challenges. Springer, Singapore, 2020.

36. Rout, S. S., Mohapatra, H., Nayak, R. K., Tripathy, R., Bhise, D., Patil, S. P., and Rath, A. K. Smart water solution for monitoring of water usage based on weather condition. International Journal of Engineering and Technical Research, 8 (2020), 5335-5343.

37. Mohapatra, H. and Rath, A. K. IoT-based smart water. IOT Technologies in Smart-Cities: From Sensors to Big Data, Security and Trust (2020), 63.

38. Mohapatra, H. and Rath, A. K. Nub less sensor based smart water tap for preventing water loss at public stand posts. In Microwave Theories and Techniques in Wireless Communications (MTTW'20), IEEE (2020), 145-150.

39. Nirgude, V. N., Nirgude, V. N., Mahapatra, H., and Shivarkar, S. A. Face recognition system using principal component analysis \& linear discriminant analysis method simultaneously with 3d morphable model and neural network BPNN method. Global Journal of Advanced Engineering Technologies and Sciences, 4 (2017), 1-6. 